\title{
Pain and Plasticity: Is Chronic Pain Always Associated with Somatosensory Cortex Activity and Reorganization?
}

\author{
Sylvia M. Gustin, ${ }^{1}$ Chris C. Peck, ${ }^{2}$ Lukas B. Cheney, ${ }^{1}$ Paul M. Macey, ${ }^{3}$ Greg M. Murray, ${ }^{2}$ and Luke A. Henderson ${ }^{1}$ \\ ${ }^{1}$ Discipline of Anatomy and Histology, University of Sydney, Sydney, New South Wales 2006, Australia, ${ }^{2}$ Faculty of Dentistry, University of Sydney, Sydney, \\ New South Wales 2006, Australia, ${ }^{3}$ Department of Neurobiology, University of California at Los Angeles, Los Angeles, California 90024
}

\begin{abstract}
The somatosensory cortex remodels in response to sensory deprivation, with regions deprived of input invaded by neighboring representations. The degree of cortical reorganization is correlated with ongoing pain intensity, which has led to the assumption that chronic pain conditions are invariably associated with somatosensory cortex reorganization. Because the presentation and etiology of chronic pain vary, we determined whether cortical changes in human subjects are similar for differing pain types. Using functional and anatomical magnetic resonance imaging, we found that, while human patients with neuropathic pain displayed cortical reorganization and changes in somatosensory cortex activity, patients with non-neuropathic chronic pain did not. Furthermore, cortical reorganization in neuropathic pain patients was associated with changes in regional anatomy. These data, by showing that pain per se is not associated with cortical plasticity, suggest that treatments aimed at reversing cortical reorganization should only be considered for use in patients with certain types of chronic pain.
\end{abstract}

\section{Introduction}

A fundamental property of the human CNS is its ability to remodel. Limb amputation or complete sensory loss results in cortical functional plasticity, in which the region deprived of sensory input is now activated by stimulation of neighboring body regions; that is, the affected region displays functional reorganization. This functional reorganization appears to occur from both the unmasking of latent synapses and the growth of new connections (Merzenich et al., 1984; Birbaumer et al., 1997). However, the consequences of such changes are varied, as cortical plasticity can result in beneficial or detrimental behavioral effects. For example, environmental enrichment can induce anatomical plasticity, such as altered dendritic ramification and dendritic spine numbers, which is associated with increased learning and memory (Rampon et al., 2000; van Praag et al., 2000). In contrast, cortical plasticity as revealed by primary somatosensory cortex (S1) reorganization in conditions of complete sensory deprivation is associated with ongoing pain (Flor et al., 1995; Wrigley et al., 2009). Indeed, a significant relationship between the degree of S1 reorganization and pain intensity also occurs in nondeafferented pain conditions, such as lower back pain (Flor et al., 1997) and complex regional pain syndrome (Maihöfner et al., 2003).

Received April 9, 2012; revised Aug. 16, 2012; accepted Aug. 16, 2012.

Author contributions: S.M.G., C.C.P., G.M.M., and L.A.H. designed research; S.M.G., C.C.P., L.B.C., G.M.M., and L.A.H. performed research; S.M.G., L.B.C., P.M.M., and L.A.H. analyzed data; S.M.G., C.C.P., P.M.M., G.M.M., and L.A.H. wrote the paper.

This work was supported by the National Health and Medical Research Council of Australia, Grant\#1032072, and the Australian Dental Research Foundation, Inc. We thank the many volunteers in this study

The authors declare no competing financial interests.

Correspondence should be addressed to Luke A. Henderson, Discipline of Anatomy and Histology, University of Sydney, Sydney, New South Wales 2006, Australia. E-mail: Iukeh@anatomy.usyd.edu.au.

DOI:10.1523/JNEUROSCI.1733-12.2012

Copyright $\odot 2012$ the authors $\quad 0270-6474 / 12 / 3214874-11 \$ 15.00 / 0$
An increasing number of scientists hold the view that chronic pain is invariably associated with $\mathrm{S} 1$ functional reorganization (Vartiainen et al., 2009; Moseley and Flor, 2012). That is, increased S1 activity both results in the perception of pain and drives a change in the relevant cortical representation (May, 2008). However, few studies have reported increased S1 activity in chronic pain conditions and, furthermore, some evidence suggests that chronic pain following limb amputation and complete spinal cord injury is associated with shrinkage, not expansion, of the S1 representation (Lenz et al., 1987; Garraghty et al., 1991; Flor et al., 1995; Birbaumer et al., 1997; Wrigley et al., 2009; Klug et al., 2011). If chronic pain induces or is caused by cortical plastic changes, then other non-neuropathic chronic pain conditions should also be associated with cortical reorganization and with $\mathrm{S} 1$ activity changes related to the degree of cortical plasticity. Whether all forms of chronic pain are associated with S1 reorganization is of significant clinical interest because novel treatment regimes aimed at altering cortical reorganization and consequently ongoing pain are being developed (Barker et al., 2008).

The aim of this investigation is first to use functional magnetic resonance imaging ( $\mathrm{PMRI}$ ) to determine whether S1 reorganization occurs in both neuropathic and non-neuropathic pain conditions. Second, we will use diffusion tensor imaging (DTI) and quantitative arterial spin labeling (QASL) to determine whether any changes in $\mathrm{S} 1$ organization are related to changes in cortical anatomy and ongoing brain activity, respectively. If all chronic pain conditions are associated with cortical plasticity and the degree of plasticity is positively correlated to pain intensity, regional S1 microstructure, and ongoing activity, an individual's ability to form new connections may predispose that person to developing changes in the activity of pain-encoding areas and ultimately chronic pain. 


\section{Materials and Methods}

\section{Subjects}

Fifteen patients with painful trigeminal neuropathy [PTN; 12 females; mean age, $50 \pm 2$ years $( \pm$ SEM) $], 17$ patients with painful temporomandibular disorder (TMD; 13 females; mean age, $44 \pm 3$ years), and 53 healthy controls without facial pain ( 27 females; mean age, $41 \pm 2$ years) were recruited for the study at the Faculty of Dentistry, Westmead Hospital, University of Sydney, Australia. All TMD patients were examined and diagnosed using the Research Diagnostic Criteria for TMD (Dworkin and LeResche, 1992) and all PTN patients were diagnosed using the Liverpool Criteria (Nurmikko and Eldridge, 2001). TMD is thought to result primarily from the activation of peripheral nociceptors (Sarlani and Greenspan, 2005; Ro, 2008; Manfredini and Nardini, 2010), although it may also contain a small neuropathic component. In contrast, PTN is commonly associated with a structural lesion or systematic disease and may result from direct trauma to the nerve. In contrast, trigeminal neuralgias are thought to result from neurovascular compression. Importantly, while trigeminal neuralgia is characterized by intermittent short episodes of sharp and shooting pain, PTN is characterized by episodic sharp and shooting pain combined with constant or long episodes of background aching and burning pain. In this manuscript, we refer to PTN as neuropathic and TMD as non-neuropathic because it has a relatively small neuropathic component if any at all. Informed written consent was obtained for all procedures and the study was approved by Institutional Human Research Ethics Committees of the University of Sydney.

\section{Psychophysical measures}

To assess the intensity of facial pain, each PTN and TMD subject rated his or her ongoing pain intensity immediately before the MRI scanning session with a vertical pencil stroke on a $10 \mathrm{~cm}$ horizontal line [visual analog scale (VAS); $0 \mathrm{~cm}=$ "no pain" to $10 \mathrm{~cm}=$ "maximum imaginable pain"]. In addition, each subject was asked to indicate, on $10 \mathrm{~cm}$ VASs his or her pain three times a day for the week before the scanning session (pain diary). These pain diary values were averaged to provide an indication of each subject's chronic pain rating. Significant differences in pain intensities and durations between pain groups were assessed using the nonparametric Mann-Whitney $U$ with $p<0.5$ considered significant. Each TMD and PTN subject also completed a McGill Pain Questionnaire (MPQ) (Melzack, 1975). The MPQ consists of a series of numerically graded adjectives in categories related to the sensory, affective, and motivational aspects of pain. Subjects were instructed to select a single item in each category if relevant to their present pain. Significant differences in the frequency of sensory descriptors chosen by each pain group were assessed using a $\chi^{2}$ test with $p<0.05$ considered significant. Finally, each patient also drew a distribution map of his or her ongoing pain onto a standard drawing of the face.

\section{MRI acquisition}

Two separate scanning series were performed. During the first series, subjects lay supine on the bed of a 3 T MRI scanner (Intera, Philips) with their head immobilized in a tight-fitting head coil. In 26 control and all PTN and TMD patients, multiple series of 130 gradient echo echo-planar fMRI image volumes using blood oxygen level-dependent contrast were collected. Each image volume contained 43 axial slices covering the entire brain (voxel, $1.95 \times 1.95 \times 3.00 \mathrm{~mm}$ thick; repetition time, $3000 \mathrm{~ms}$; echo time, $40 \mathrm{~ms}$ ). During each fMRI series, the lateral part of the lower lip, the distal pad of the little finger, or the distal pad of the thumb was brushed with a plastic brush at $\sim 2$ strokes/s. This stimulation paradigm was performed for a period of $10 \mathrm{fMRI}$ volumes $(30 \mathrm{~s})$ following a baseline period of $10 \mathrm{fMRI}$ volumes $(30 \mathrm{~s})$. This was repeated a further five times for a total of six stimulation and seven baseline periods. In control subjects, only the right side of the body was brushed. In all TMD and PTN subjects, the side ipsilateral to the highest ongoing pain was brushed. In addition, in six of seven PTN patients that displayed unilateral orofacial pain, the side contralateral to the pain was also brushed. A 3D T1-weighted image set was also collected (voxel size, $0.90 \times 0.90 \times 0.90 \mathrm{~mm}$ ).

In 21 of the 26 controls and all TMD and PTN patients, using a singleshot multisection spin-echo echo-planar pulse sequence, four high- resolution DTI sets covering the entire brain were collected (repetition time, $8788 \mathrm{~ms}$; flip angle, $90^{\circ}$; matrix size, $112 \times 112$; field of view, $224 \times$ $224 \mathrm{~mm}$; slice thickness, $2.5 \mathrm{~mm}$; 55 axial slices). For each slice, diffusion gradients were applied along 32 independent orientations with $b=1000$ $\mathrm{s} / \mathrm{mm}^{2}$ after the acquisition of $b=0 \mathrm{~s} / \mathrm{mm}^{2}$ (b0) images. Four DTI series were collected separately for subsequent averaging.

During the second scanning series, in $10 \mathrm{TMD}, 12 \mathrm{PTN}$, and 6 controls who participated in the first scanning session, as well as an additional 28 control subjects, whole-brain cerebral blood flow (CBF) maps were acquired using QASL. The pulsed ASL version, called quasar, was used for this study and the sequence and subsequent CBF quantification has been described in details previously (Petersen et al., 2006, 2010). In short, the sequence is a multislice, multiple time-points-capable sequence based on pulsed ASL and a Look-Locker readout. In addition, the sequence acquires data with and without vascular crushers (velocity cutoff, $4 \mathrm{~cm} / \mathrm{s}$ ), which enables the estimation of the arterial input function on a regional level. CBF is subsequently estimated by model-free deconvolution, similar to what is done for gadolinium perfusion scans (Petersen et al., 2006). By adding a few dynamics at a lower flip angle, correct B1, T1, and M0 of tissue can be obtained, which are subsequently used for CBF quantification (Petersen et al., 2010). General scan parameters were as follows: TR, $4000 \mathrm{~ms}$; TE, $23 \mathrm{~ms} ; \Delta \mathrm{TI}, 300 \mathrm{~ms}$, TI1, $40 \mathrm{~ms} ; 13$ inversion times, $40-$ $3640 \mathrm{~ms}$; matrix, $64 \times 64$; number of slices, 7 ; slice thickness, $6 \mathrm{~mm}$; gap, $2 \mathrm{~mm}$; field of view, $240 \times 240$; flip angle; $35 / 11.7^{\circ}$; SENSE (sensitivity encoding), $2.5 ; 84$ averages ( 48 with crusher, $4 \mathrm{~cm} / \mathrm{s} ; 24$ without crushers; 12 low flip angle), all implemented in a single sequence with a scan duration of 5 min $52 \mathrm{~s}$.

\section{MRI analysis}

fMRI analysis. Using SPM8 (Friston et al., 1995) and FreeSurfer (Pleger et al., 2004) software, each subject's T1-weighted anatomical image set was spatially normalized to the Montreal Neurological Institute (MNI) template and segmented into gray, white, and CSF images. Using the gray-matter images, the left and right postcentral gyrus (S1) were automatically isolated in each individual subject. In each subject, the fMRI image sets were realigned and then coregistered to their own spatially normalized T1-weighted anatomical image set. The isolated postcentral gyrus obtained from the $\mathrm{T} 1$ anatomical image set was then used to isolate the postcentral gyrus in each individual subject's fMRI image sets. Visual inspection revealed highly accurate postcentral gyrus isolation in each subject and resulted in the fMRI analysis being restricted to each individual's gray matter of his or her S1. Global signal drifts in fMRI image sets were removed using the detrending method described by Macey and colleagues (2004) and images were finally spatially smoothed using a $6 \mathrm{~mm}$ full-width-at-half-maximum (FWHM) Gaussian filter. Significant increases in fMRI signal intensity were determined using a repeated box-car model convolved with hemodynamic delay function. Those voxels within the contralateral S1 that displayed significant increases in signal intensity (random effects, $p<0.05$ familywise error corrected for multiple comparisons; minimum cluster size, 10 voxels) were determined. In each subject, the percentage change (relative to baseline periods) in signal intensity over time of a $4 \mathrm{~mm}$ sphere centered around the maximally activated voxel was determined and significant differences between groups assessed (2-tailed $t$ test, $p<0.05$ ).

Euclidean distances (EDs) were then calculated between a standard anatomical point in each individual subject (the point at which the central sulcus meets the longitudinal fissure at the dorsal aspect of the brain) and the maximally activated voxel within the contralateral S1 during each brushing paradigm. This procedure has been described in detail previously (Wrigley et al., 2009). A Mann-Whitney $U$ test was used to determine significant differences (SPSS software, IBM; $p<0.05$ ) in the ED of $\mathrm{S} 1$ activations between control, TMD, and PTN subjects. In addition, a one-tailed Pearson's correlation test was used to determine significant $(p<0.05)$ correlations between these EDs and the mean pain intensity scores on the day of scanning.

DTI analysis. The diffusion-weighted images were motion corrected, coregistered to one another, and averaged to increase signal-to-noise ratio. Using the 32 directions and b0 images, the diffusion tensor was then calculated using the method proposed by Basser and Pierpaoli 
Table 1. Characteristics of PTN subjects

\begin{tabular}{|c|c|c|c|c|c|c|c|c|}
\hline Subject & Age & Gender & Pain (years) & Site & $\begin{array}{l}1 \text { week pain } \\
\text { diary (VAS) }\end{array}$ & $\begin{array}{l}\text { Pain prior to } \\
\text { scan (VAS) }\end{array}$ & Analgesic medication & Pain outside orofacial region \\
\hline 1 & 51 & $M$ & 3.5 & Right & 1.6 & 1.7 & Amitriptyline & Lower back \\
\hline 2 & 48 & $\mathrm{~F}$ & 9.0 & Bilateral & 3.3 & 2.5 & Gabapentin & Neck \\
\hline 3 & 47 & $\mathrm{~F}$ & 5.0 & Left & 1.1 & 1.9 & None & \\
\hline 4 & 53 & $\mathrm{~F}$ & 2.5 & Right & 1.5 & 1.1 & $\begin{array}{l}\text { None } \\
\text { Gabapentin } \\
\text { Oxycodone }\end{array}$ & \\
\hline 5 & 52 & $\mathrm{~F}$ & 1.5 & Bilateral & 6.9 & 7.0 & $\begin{array}{l}\text { Paracetamol } \\
\text { Amitriptyline } \\
\text { Gabapentin } \\
\text { Oxycodone Hydrochloride }\end{array}$ & Lower back \\
\hline 6 & 55 & $\mathrm{~F}$ & 2.0 & Left & 5.2 & 6.7 & Paracetamol & \\
\hline 7 & 46 & M & 9.0 & Bilateral & 3.1 & 1.8 & $\begin{array}{l}\text { None } \\
\text { Diazepam } \\
\text { Paracetamol }\end{array}$ & \\
\hline 8 & 46 & $\mathrm{~F}$ & 3.0 & Bilateral & 6.5 & 5.2 & $\begin{array}{l}\text { Ibuprofen (as needed) } \\
\text { Carbamazepine }\end{array}$ & \\
\hline 9 & 42 & $\mathrm{~F}$ & 11.0 & Bilateral & 4.8 & 4.0 & Paracetamol & Neck/shoulders, lower back \\
\hline 10 & 48 & $\mathrm{~F}$ & 1.25 & Bilateral & 3.1 & 2.8 & $\begin{array}{l}\text { Gabapentin } \\
\text { Pregabalin }\end{array}$ & Neck/shoulders, arms/hands \\
\hline 11 & 34 & M & 5.0 & Bilateral & 3.5 & 1.9 & $\begin{array}{l}\text { Nortriptyline } \\
\text { Paracetamol }\end{array}$ & \\
\hline 12 & 59 & $\mathrm{~F}$ & 5.0 & Left & 3.3 & 2.2 & Ibuprofen & Lower back \\
\hline 13 & 54 & $\mathrm{~F}$ & 2.0 & Right & 2.0 & 5.7 & Amitriptyline & Wrist (right) \\
\hline 14 & 44 & $\mathrm{~F}$ & 6.5 & Bilateral & 6.4 & 6.0 & None & \\
\hline 15 & 40 & $\mathrm{~F}$ & 3.5 & Bilateral & 4.0 & 4.0 & Carbamazepine & Neck, hip (right) \\
\hline Mean ( \pm SEM) & $50( \pm 2)$ & & $4.7( \pm 0.8)$ & & $3.8( \pm 0.5)$ & $3.6( \pm 0.5)$ & & \\
\hline
\end{tabular}

All patients fulfilled the criteria for PTN according to the Liverpool Criteria (Nurmikko and Eldridge, 2001).

(1996). Once the elements of diffusion tensor were calculated, fractional anisotropy (FA) maps were derived and the images spatially normalized and smoothed (FWHM, $6 \mathrm{~mm}$ ). Significant differences in FA within the contralateral S1 between control, TMD, and PTN subjects were determined (random effects, $p<0.05$ familywise error corrected for multiple comparisons; minimum cluster size, 10 voxels; age and gender as nuisance variables). In addition, FA values within the contralateral S1 that were significantly correlated to the degree of cortical reorganization in PTN subjects were determined $(r>0.06$, age and gender as nuisance variables). The degree of cortical reorganization in individual PTN subjects was calculated by subtraction of the mean ED during brushing of the thumb in healthy subjects and the individual ED in PTN subjects. Significant FA clusters were then overlaid onto an individual's T1-weighted anatomical image. For each cluster, the significance of the relationship between FA and degree of S1 reorganization was determined $(p<0.05)$. Furthermore, these significant clusters were overlaid onto the FA images of control and TMD subjects and the mean ( \pm SEM) FA values calculated and compared between groups (2-tailed $t$ test, $p<0.05$ ).

In the $\mathrm{S} 1$ region where the degree of reorganization significantly correlated to FA, the directional nature of structure was indicated on a polar plot with a vector corresponding to the direction of the principal eigenvector of the diffusion tensor. This vector indicates the predominant direction of water diffusion, and therefore is an indirect measure of axonal direction. To better understand the nature of those structural changes, we calculated the mean direction across voxels in regions that showed the greatest structural differences. We also indicated variation by displaying vectors of the mean plus or minus the median absolute deviation (in lieu of SD, which is complex for vectors) as well as individual voxel principal eigenvectors.

QASL analysis. We opened QASL images using custom software developed by Petersen and colleagues (2006) and created CBF maps. In addition, we created from the CBF maps anatomical (gray/white) image sets. These anatomical images were then coregistered to the T1-weighted anatomical image set collected at the same slice locations and the resulting parameters applied to the CBF maps. The T1-weighted anatomical images were then normalized to the standard MNI template and the normalization parameters applied to the CBF maps.
Significant differences in CBF within the contralateral S1 between control, TMD, and PTN subjects were determined (random effects, $p<0.05$ familywise error corrected for multiple comparisons; minimum cluster size, 10 voxels; age and gender as nuisance variables). In addition, CBF values within the contralateral S1 that were significantly correlated to the degree of cortical reorganization in PTN subjects (mean ED during brushing of the thumb in healthy subjects and the individual ED in PTN subjects) were determined ( $r>0.06$; age and gender as nuisance variables). Significant CBF clusters were then overlaid onto an individual's T1-weighted anatomical image. For each cluster, the significance of the relationship between CBF and degree of S1 reorganization was determined $(p<0.05)$. Furthermore, these significant clusters were overlaid onto the CBF images of control and TMD subjects and the mean ( \pm SEM) CBF values calculated and compared between groups (2-tailed $t$ test, $p<0.05$ ).

\section{Results}

\section{Pain distribution and quality}

In most pain patients $(n=22)$, their orofacial pain was restricted to areas innervated by the maxillary and mandibular divisions of the trigeminal nerve and was similar in intensity and duration in both groups (intensity TMD, $4.2 \pm 0.5$; PTN, $3.8 \pm 0.5 ; p>0.05$; duration: TMD, $4.2 \pm 0.5$ years; PTN, $3.8 \pm 0.5$ years; $p>0.05)$. Eight PTN and 14 TMD patients had bilateral orofacial pain where as the remaining 7 PTN and 3 TMD patients had orofacial pain restricted to one side (Tables $1,2)$. In addition, 8 PTN patients and 12 TMD patients also described low-level pain outside the orofacial region. In TMD patients, pain often also occurred in the neck and shoulders, whereas in PTN patients, it occurred at disparate body sites. There was no significant difference in pain duration in the PTN compared with TMD groups $(p=0.13)$.

Using the MPQ to assess the sensory aspects of pain, it was found that descriptions PTN patients had for their pain differed from the descriptions of pain offered by the TMD patients. PTN 
Table 2. Characteristics of TMD subjects

\begin{tabular}{|c|c|c|c|c|c|c|c|c|}
\hline Subject & Age & Gender & Pain (years) & Site & $\begin{array}{l}1 \text { week pain } \\
\text { diary (VAS) }\end{array}$ & $\begin{array}{l}\text { Pain prior to } \\
\text { scan (VAS) }\end{array}$ & Analgesic medication & Pain outside orofacial region \\
\hline 1 & 38 & $\mathrm{~F}$ & 6.0 & Right & 1.8 & 4.7 & Paracetamol & Shoulder (left) \\
\hline 2 & 59 & M & 20.0 & Bilateral & 5.9 & 6.0 & $\begin{array}{l}\text { Amitriptyline } \\
\text { Venlafaxine }\end{array}$ & \\
\hline 3 & 59 & $\mathrm{~F}$ & 30.0 & Bilateral & 7.9 & 7.6 & Co-codamol & Neck/shoulders, lower back \\
\hline 4 & 47 & M & 20.0 & Bilateral & 2.9 & 2.7 & $\begin{array}{l}\text { Paracetamol (as needed) } \\
\text { Diazepam }\end{array}$ & \\
\hline 5 & 62 & $\mathrm{~F}$ & 5.0 & Bilateral & 5.4 & 3.9 & Paracetamol (as needed) & Neck/shoulders, lower back \\
\hline 6 & 45 & $\mathrm{~F}$ & 2.0 & Bilateral & 1.5 & 1.1 & None & Neck/shoulders, lower back \\
\hline 7 & 37 & M & 6.0 & Bilateral & 7.3 & 7.0 & Botox & \\
\hline 8 & 41 & $\mathrm{~F}$ & 4.0 & Right & 1.4 & 0.5 & None & \\
\hline 9 & 50 & $\mathrm{~F}$ & 46.0 & Bilateral & 4.3 & 6.5 & $\begin{array}{l}\text { Diclofenac } \\
\text { Oxycodone (as needed) } \\
\text { Paracetamol } \\
\text { Co-codamol (as needed) }\end{array}$ & Neck/shoulders, lower back \\
\hline 10 & 45 & $\mathrm{~F}$ & 5.5 & Bilateral & 4.1 & 7.3 & Doxylamine succinate and codeine & Neck/shoulders, lower back \\
\hline 11 & 33 & $\mathrm{~F}$ & 15.0 & Right & 5.9 & 5.5 & Doxylamine succinate and codeine (as needed) & Neck/shoulders, Arms/hands, lower back \\
\hline 12 & 28 & M & 1.5 & Bilateral & 5.8 & 6.7 & $\begin{array}{l}\text { Ibuprofen (as needed) } \\
\text { Amitriptyline } \\
\text { Co-codamol }\end{array}$ & Neck/shoulders, arms/hands, lower back \\
\hline 13 & 24 & $\mathrm{~F}$ & 7.0 & Bilateral & 5.5 & 5.9 & Sumatriptan & Neck/shoulders \\
\hline 14 & 66 & $\mathrm{~F}$ & 5.0 & Bilateral & 3.1 & 1.1 & None & Neck/shoulder, arms/hands, lower back \\
\hline 15 & 50 & $\mathrm{~F}$ & 3.0 & Bilateral & 2.7 & 2.0 & None & Neck/shoulders \\
\hline 16 & 37 & $\mathrm{~F}$ & 4.0 & Bilateral & 2.2 & 5.5 & $\begin{array}{l}\text { Ibuprofen (as needed) } \\
\text { Ibuprofen }\end{array}$ & Neck/shoulders, lower back \\
\hline 17 & 30 & $\mathrm{~F}$ & 2.0 & Bilateral & 3.5 & 1.7 & Paracetamol & \\
\hline Mean ( \pm SEM) & $44( \pm 3)$ & & $10.7( \pm 2.9)$ & & $4.2( \pm 0.5)$ & $4.5( \pm 0.6)$ & & \\
\hline
\end{tabular}

All TMD patients fulfilled the criteria for TMD according to the Research Diagnostic Criteria for TMD (Dworkin and LeResche, 1992).

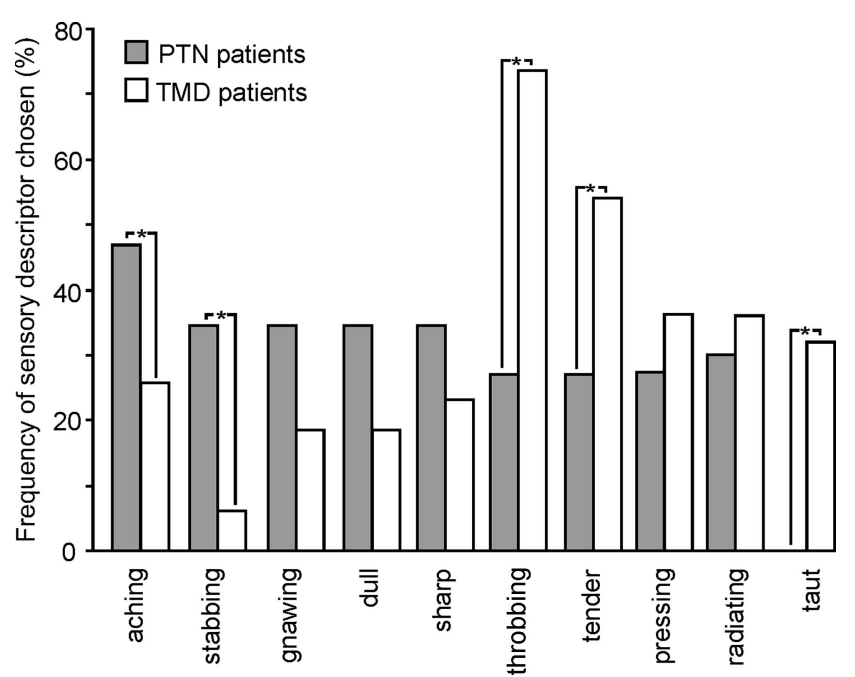

Figure 1. Sensory descriptors. Frequency (percentage of subjects) of the five most common pain descriptors selected from the sensory component of the MPQ by patients with PTN (gray bars) or TMD (white bars). Asterisk indicates significant differences between groups $(p<0.05)$.

patients most often described their pain as "aching," "stabbing," and "sharp," whereas TMD patients described their pain as "throbbing," "pressing," and "tender" (Fig. 1). Furthermore, PTN patients chose the descriptors "aching" and "stabbing" significantly more often than TMD patients, and TMD patients in describing their pain chose "throbbing, "tender," and "taut" significantly more often than PTN patients did.

\section{S1 functional organization}

In all subjects, innocuous brushing of the lip, thumb, and little finger resulted in a lateral-to-medial pattern of activation within the contralateral postcentral gyrus (S1), consistent with the well described somatosensory homunculus. In all groups, brushing was perceived as innocuous. Although the general organization of S1 activation was similar in all three groups, there were significant differences in the precise locations of S1 activations (Table 3). In control subjects, innocuous brushing of the lip, thumb, and little finger resulted in regional activations within the contralateral postcentral gyrus that were centered (mean \pm SEM) $71.4 \pm$ $1.1,64.6 \pm 1.5$, and $59.6 \pm 2.4 \mathrm{~mm}$ from the vertex of the central sulcus, respectively. A similar set of activations also occurred in TMD patients (lip, $71.9 \pm 1.4 \mathrm{~mm}$; thumb, $64.5 \pm 2.0 \mathrm{~mm}$; little finger, $61.8 \pm 3.3 \mathrm{~mm} ; p>0.05$; 2-tailed, 2-sample $t$ test). That is, TMD patients did not display S1 functional reorganization (Fig. 2). In direct contrast, in PTN subjects, although the location of the lip activation was not significantly different from that of controls, the locations of the thumb and little finger activations were shifted mediodorsally compared with controls (lip, $69.8 \pm 1.6$ $\mathrm{mm}$; thumb, $55.4 \pm 2.7 \mathrm{~mm}$; little finger, $53.0 \pm 2.8 \mathrm{~mm} ; p<$ 0.05 ; 2-tailed, 2-sample $t$ test for thumb and little finger). That is, PTN patients displayed a significant $S 1$ functional reorganization in which the area representing the ongoing pain (lip) appeared to expand. Furthermore, in six PTN patients with unilateral orofacial pain, brushing on the side of the body contralateral to the ongoing pain revealed no significant change in ipsilateral S1 organization relative to controls (lip, $65.4 \pm 3.2 \mathrm{~mm}$; thumb, $57.6 \pm 2.1 \mathrm{~mm}$; little finger, $56.7 \pm 1.7 \mathrm{~mm} ; p<0.05$, 2-tailed, 2 -sample $t$ test for thumb and little finger). Finally, although PTN patients displayed significant $\mathrm{S} 1$ reorganization, there was no significant relationship between the change in locations during thumb or little finger brushing and pain intensity during scanning (thumb, $r=0.22$; little finger, $r=-0.30$; $p>0.05$ ), pain diary (thumb, $r=0.11$; little finger, $r=0.01 ; p>0.05$ ), or pain duration (thumb, $r=-0.48$; little finger, $r=-0.14 ; p>0.05$ ). 
Table 3. Mean ( \pm SEM) MNI co-ordinates of maximally activated voxels in the contralateral S1 during innocuous brushing of the lip, thumb, and little finger in control, TMD, and PTN subjects

\begin{tabular}{|c|c|c|c|c|c|c|c|c|c|c|c|c|}
\hline & \multicolumn{4}{|l|}{ Controls } & \multicolumn{4}{|l|}{ TMD } & \multicolumn{4}{|l|}{ PTN } \\
\hline & $x$ & $y$ & $Z$ & ED & $x$ & $y$ & $z$ & ED & $x$ & $y$ & $z$ & $E D$ \\
\hline Lip & $-58.3 \pm 0.7$ & $-15.7 \pm 0.7$ & $40.3 \pm 0.7$ & $71.4 \pm 1.1$ & $-57.4 \pm 0.3$ & $-14.7 \pm 0.9$ & $38.9 \pm 0.7$ & $71.9 \pm 1.4$ & $-56.8 \pm 1.2$ & $-15.4 \pm 1.4$ & $40.1 \pm 2.1$ & $69.8 \pm 1.6$ \\
\hline Thumb & $-55.3 \pm 0.9$ & $-21.1 \pm 2.0$ & $47.8 \pm 1.1$ & $64.6 \pm 1.5$ & $-53.9 \pm 1.4$ & $-17.5 \pm 1.3$ & $46.9 \pm 2.7$ & $64.5 \pm 2.0$ & $-48.9 \pm 2.2$ & $-19.3 \pm 1.6$ & $53.9 \pm 1.6$ & $55.4 \pm 2.7$ \\
\hline Little finger & $-52.0 \pm 1.5$ & $-23.7 \pm 1.3$ & $49.4 \pm 1.9$ & $59.6 \pm 2.4$ & $-52.4 \pm 1.5$ & $-22.0 \pm 2.2$ & $49.8 \pm 2.8$ & $61.8 \pm 3.3$ & $-48.0 \pm 2.2$ & $-22.4 \pm 1.3$ & $53.0 \pm 2.4$ & $53.0 \pm 3.0$ \\
\hline
\end{tabular}

\section{SI organization and chronic pain}

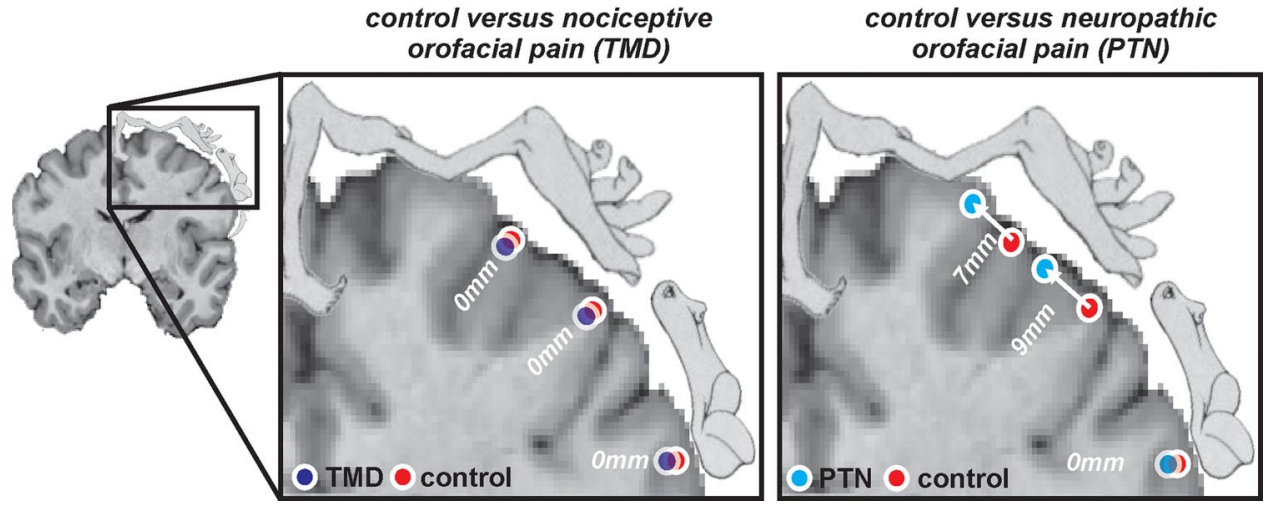

Figure 2. Primary somatosensory organization. Plots of mean ( \pm SEM) Euclidean distances in millimeters from the point at which the central sulcus meets the midline to the maximally activated voxel in the contralateral S1 during innocuous brushing of the lip, thumb, and little finger in control (red), painful TMD (dark blue), and PTN (light blue) groups. In direct contrast to PTN, TMD is not associated with a change in $\mathrm{S} 1$ organization. Asterisks indicate significant differences $(p<0.05$, Mann-Whitney $U)$ in the location of S1 activations in PTN compared with controls (red) and TMD (dark blue) subjects.

\section{S1 sensitivity}

In addition to assessing the locations of maximally activated voxels, the sensitivity of S1 to innocuous activation was also assessed. In control subjects, innocuous brushing of the lip, thumb, and little finger evoked signal intensity increases within the contralateral S1 of $\sim 1.5 \%$ (lip, $1.6 \pm 0.1 \%$; thumb, $1.8 \pm 0.2 \%$; little finger, $1.2 \pm 0.1 \%$ ) (Fig. 3). In direct contrast, in patients with either nociceptive (TMD) or neuropathic (PTN) orofacial pain, brushing evoked S1 signal intensity increases that were significantly reduced. That is, the contralateral S1 was less sensitive in patients with chronic orofacial pain (TMD: lip, $1.2 \pm 0.1 \%$; thumb, $1.1 \pm 0.1 \%$; little finger, $0.8 \pm 0.1 \%$; PTN: lip, $1.2 \pm$ $0.2 \%$; thumb, $1.2 \pm 0.2 \%$; little finger, $0.9 \pm 0.1 \%)(p<0.05$, 2-sample $t$ test).

\section{S1 reorganization and baseline activity}

To determine whether increased ongoing activity was responsible for the cortical reorganization seen in PTN patients, baseline CBF within S1 was assessed using QASL imaging. Compared with controls, PTN patients displayed a significant reduction in CBF in the region representing the area of ongoing pain (i.e., the lower face) (Fig. $4 A$, middle). In direct contrast, TMD patients did not display any difference in CBF within the contralateral S1 (Fig. $4 A$, left). Reduced $\mathrm{CBF}$ within the face representation was also seen in PTN compared with TMD patients (Fig. $4 A$, right).

In addition, we assessed whether the degree of S1 reorganization that occurred in PTN patients was related to the level of ongoing activity (Fig. $4 \mathrm{~B}$ ). We found a significant positive correlation between the degree of S1 reorganization and ongoing CBF. That is, the greater the S1 reorganization, the greater the ongoing activity. These data suggest that, in individuals with no or little reorganization, $\mathrm{CBF}$ is well below baseline levels. Meanwhile, those with high levels of reorganization have CBF levels above control levels. In contrast, we found that CBF was not significantly correlated to pain intensity during scanning $(r=0.28 ; p>$ $0.05)$, pain diary $(r=0.30 ; p>0.05)$, or pain duration $(r=0.31$; $p>0.05)$. Finally, further confirming the lack of CBF change within the contralateral S1 of TMD patients, we found that the region that displayed a significant relationship between $\mathrm{CBF}$ and $\mathrm{S} 1$ reorganization in PTN patients displayed no CBF change in TMD patients.

\section{S1 reorganization and anatomy}

To determine whether S1 reorganization was associated with a change in regional anatomy, the anatomy of contralateral S1 was assessed using diffusion-weighted imaging. Compared with controls, PTN patients displayed a significant reduction in FA, an indicator of altered microstructure, in the region representing the lower face (Fig. 5A, middle). In direct contrast, TMD patients did not display any difference in FA within the contralateral S1 (Fig. $5 A$, left). Reduced $F A$ within the face representation was also seen in PTN patients compared with TMD patients (Fig. 5A, right).

In addition, we assessed whether the degree of $\mathrm{S} 1$ reorganization that occurred in PTN patients was related to underlying brain anatomy (Fig. 5B). We found a significant positive correlation between the degree of $\mathrm{S} 1$ reorganization and FA. That is, the greater the degree of S1 reorganization, the greater the change in $\mathrm{S} 1$ anatomy. FA was not significantly correlated to pain intensity during scanning $(r=0.49 ; p>0.05)$, pain diary $(r=0.42 ; p>$ $0.05)$, or pain duration $(r=0.35 ; p>0.05)$. To further confirm the lack of FA change within the contralateral S1 of TMD patients, we found that the region displaying a significant relationship between FA and S1 reorganization in PTN patients, displayed no FA change in TMD patients. Finally, in the region of $\mathrm{S} 1$ in which the degree of reorganization was significantly correlated to FA, the directional nature of structure was indicated with 


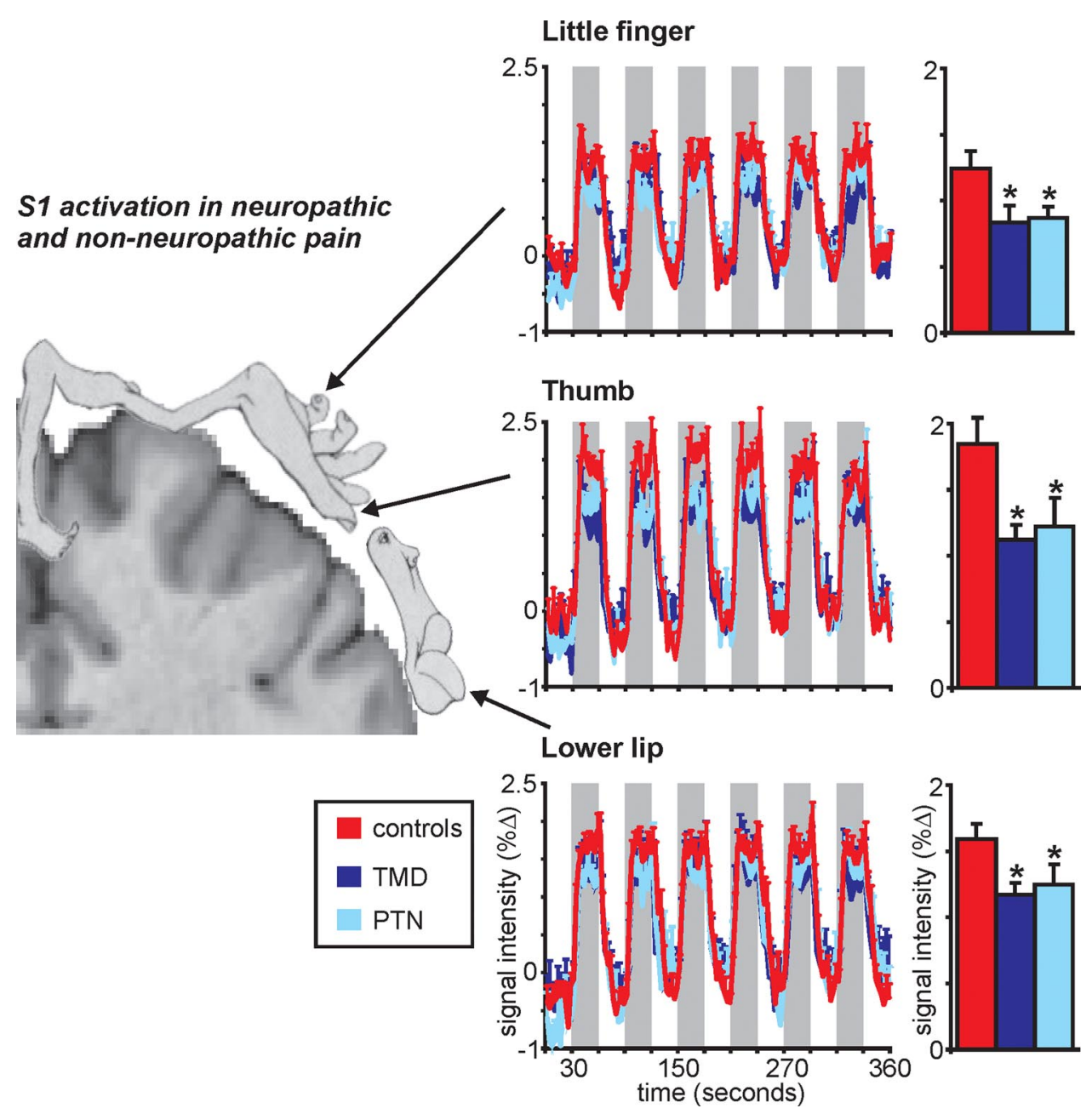

Figure 3. S1 activation during innocuous lip brushing. Signal intensity increases within the contralateral S1 during brushing of the little finger, thumb, and lower lip in control (red), painful TMD (dark blue), and PTN (light blue) groups. Plots of mean ( \pm SEM) signal intensity changes over time are derived from a $4 \mathrm{~mm}$ sphere around the maximally activated voxel in each individual subject. The vertical gray bars indicate periods of innocuous brushing. To the right are plots of mean ( \pm SEM) signal intensity change during the brushing periods compared with baseline. Although the pattern and magnitude of the signal intensity changes appear similar, there is a significant reduction in the magnitude of $S 1$ activation in both the TMD and PTN groups compared with controls ( ${ }^{*} p<$ 0.05 , 2-tailed $t$ test).

a vector indicating the directionality of the tissue organization (the principal eigenvector). Although the tissue organization in terms of directionality was similar in control and TMD patients, PTN patients showed much less consistent directional organization of tissue. That is, in all three planes, the variability of eigenvectors was considerably greater in PTN subjects compared with control and TMD subjects (Fig. 6).

\section{Discussion}

Over 25 years ago, it was shown in the owl monkey that digit amputation led to a reduction in the S1 region representing the amputated digit as neighboring representations "invaded" (Merzenich et al., 1984). Since this initial report, many investigators have reported functional reorganization of human S1 following various forms of deafferentation, such as limb amputation and complete spinal cord injury (Kaas, 1991; Flor et al., 1995, 1997; Maihöfner et al., 2003; Wrigley et al., 2009; Henderson et al., 2011). Despite these findings, close inspection reveals that, in some individuals, deafferentation is not associated with significant cortical plasticity, whereas in others, a seemingly identical injury results in a significant change in cortical representations. Surprisingly, it was revealed in the mid-1990s that the degree of S1 reorganization that occurs following deafferentation is closely associated with the development of chronic pain. That is, those individuals that display significant cortical plasticity are more likely to develop chronic pain (Flor et al., 1995).

Since this first report, many have described a significant relationship between cortical plasticity and chronic pain. Our data show that, while neuropathic orofacial pain is associated with S1 reorganization, non-neuropathic orofacial pain is not. There is some evidence to suggest that acute pain in healthy controls can result in S1 reorganization (Sörös et al., 2001). Given this, we expected that TMD patients would display increased S1 activity and a concurrent S1 reorganization. However, non-neuropathic orofacial pain, besides failing to display S1 reorganization, showed no association with changes in S1 microstructure or blood flow, two measures that were significantly altered in patients with neuropathic orofacial pain. Indeed, over the past few decades, various investigations have reported increases, decreases, or no change in S1 activity in various chronic pain conditions (Lenz et al., 1987; Garraghty et al., 1991; Klug et al., 2011). Our data suggest that $\mathrm{S} 1$ activity is not essential for the persistent perception of pain and increased S1 inputs may be required for reorganization to occur. A lack of constant S1 activity in TMD patients may be the reason why they do not display cortical reor- 

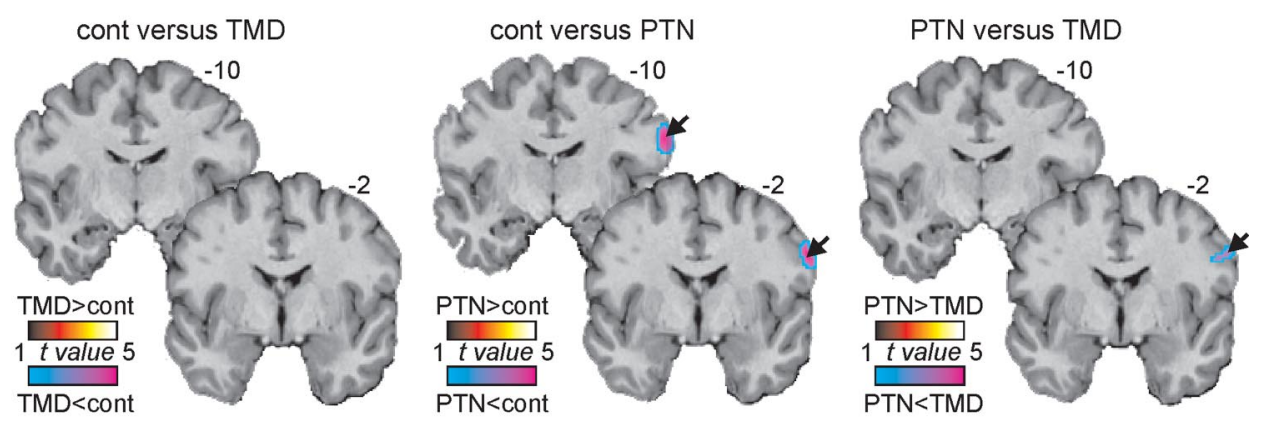

B

Baseline activity correlated to $\mathrm{S} 1$ reorganization
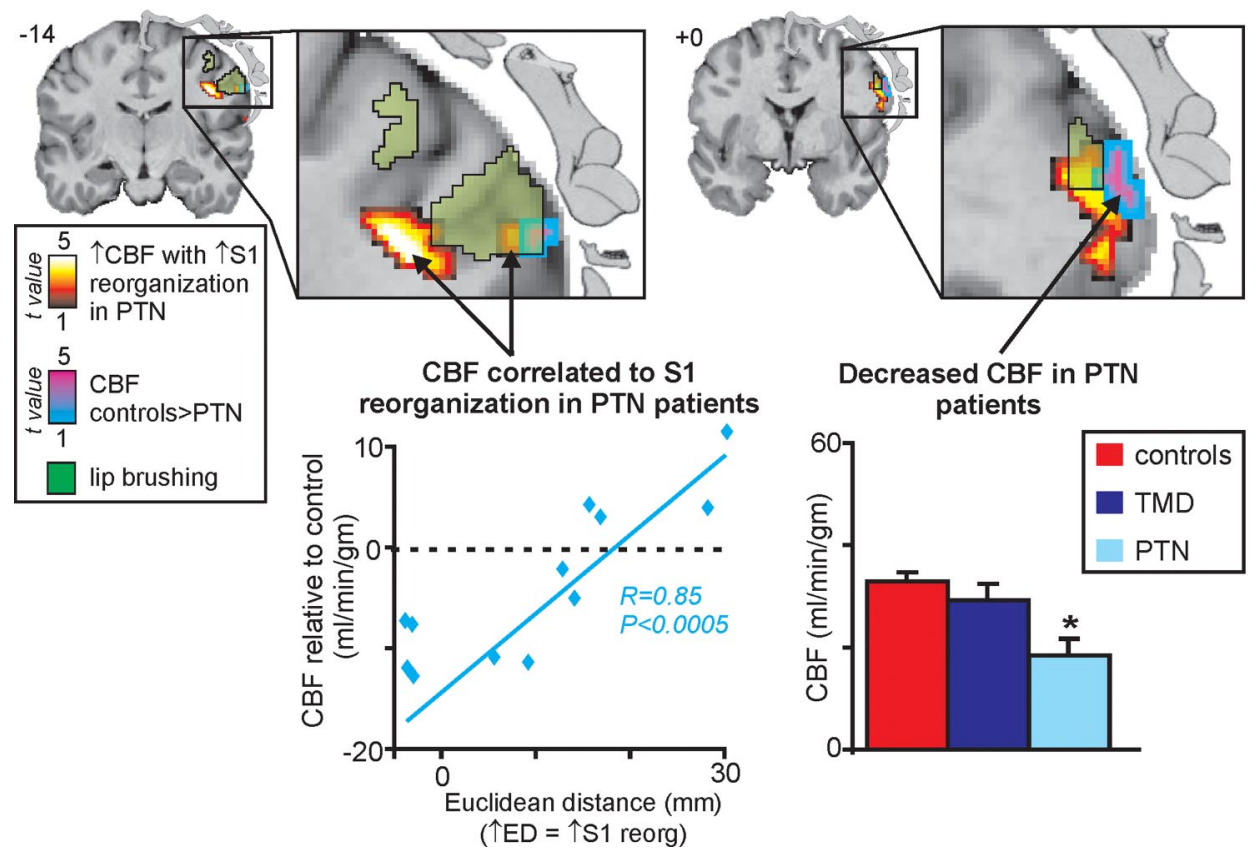

Figure 4. S1 activity and chronic pain. A, Overlays showing areas in which baseline CBF was altered in patients with painful TMD and PTN. Axial slice locations in MNI space coordinates are indicated at the top right of each image. Note that, whereas TMD patients display no significant CBF change in the contralateral S1 in comparison with controls, PTN patients display a significant CBF reduction in comparison with controls and TMD patients in the S1 region representing the lip. B, Overlays showing areas in which baseline CBF was significantly correlated with the degree of S1 reorganization in PTN patients (hot color scale). The plot of CBF (difference relative to controls) and degree of S1 reorganization on the lower left shows this significant positive correlation. Negative values indicate CBF is below control levels. The significantly correlated region overlaps with (1) the region activated by innocuous lip brushing (green shading) and (2) the region in which baseline CBF is significantly reduced in PTN patients (cool color scale). The plot of mean ( \pm SEM) CBF on the lower right shows that in the region in which CBF is reduced in PTN patients, CBF is not altered in TMD patients.

ganization. Indeed, it is possible that whether the condition is neuropathic or non-neuropathic in nature may be of little importance. Instead, the critical factor for the cortex to undergo functional reorganization may be whether there is constant S1 input.

Close inspection of previous investigations also reveals that the direction of S1 reorganization associated with pain is inconsistent. We have previously found that complete spinal cord injury is associated with contraction of the lower body S1 representation (Wrigley et al., 2009). A similar situation occurs following limb amputation (Flor et al., 1995). In direct contrast, the present study shows in a neuropathic pain condition that does not involve deafferentation, trigeminal neuropathy, the S1 representation representing the thumb moves away from that of the lip. Similarly, it has been reported that painful carpel tunnel syndrome is associated with an increased representation of the painful digits (Napadow et al., 2006) and use-dependent enlargements of cortical representations, including those in response to increased innocuous tactile stimulation, have been shown to occur in many sensory systems (Hodzic et al., 2004). In contrast, complex regional pain syndrome, a non-deafferented pain condition, is associated with contraction of the relevant cortical representation (Maihöfner et al., 2003; Pleger et al., 2004), although this condition involves significant decreases in the use of the painful region, which may itself affect S1 organization. Thus, both the extent and direction of cortical reorganization associated with different forms of chronic pain are highly variable, strongly suggesting that pain itself does not result in S1 reorganization.

Furthermore, the sensitivity of $\mathrm{S} 1$ is variable in different pain conditions. We found that both neuropathic and non- 

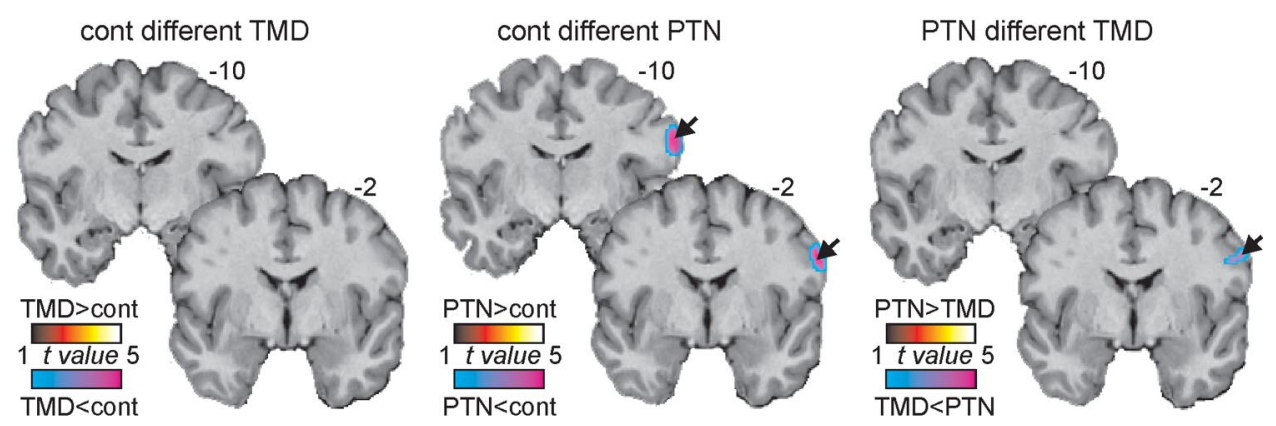

B

S1 anatomy correlated to S1 reorganization
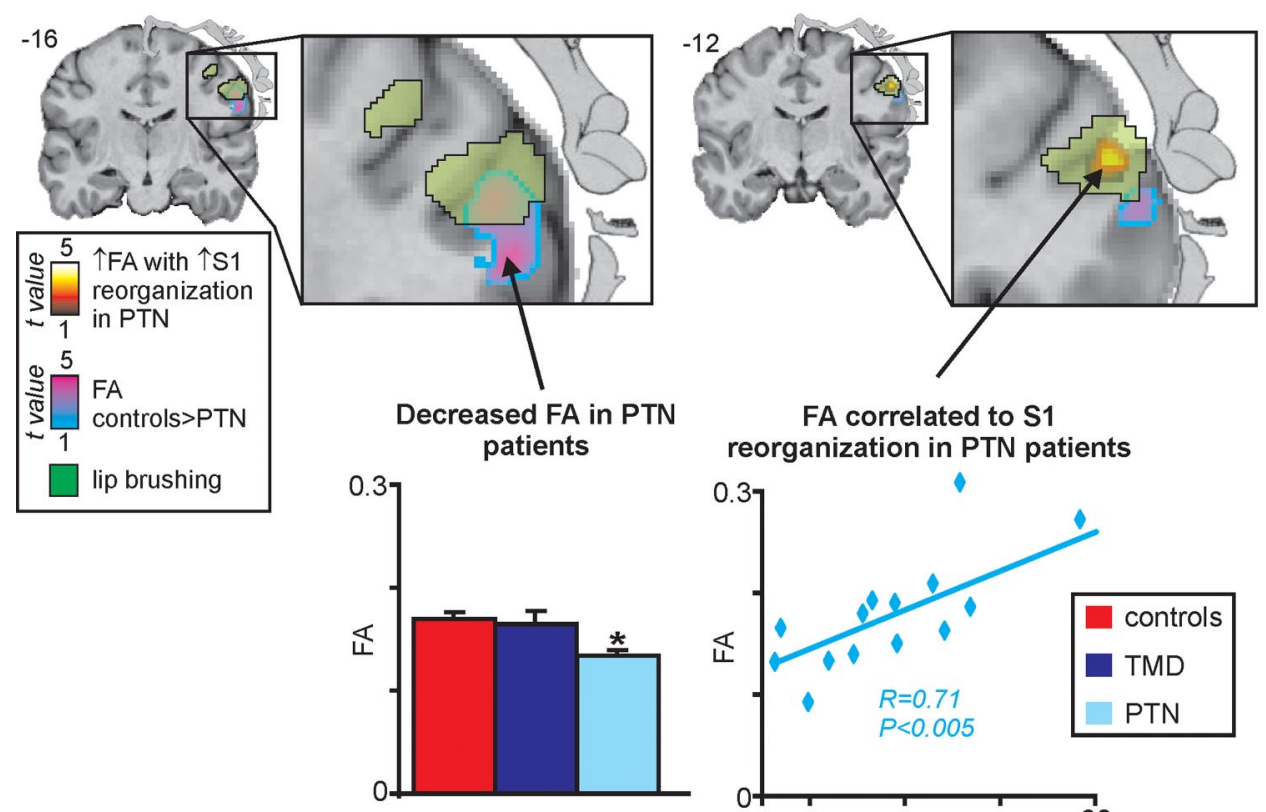

FA correlated to $\mathrm{S} 1$ reorganization in PTN patients

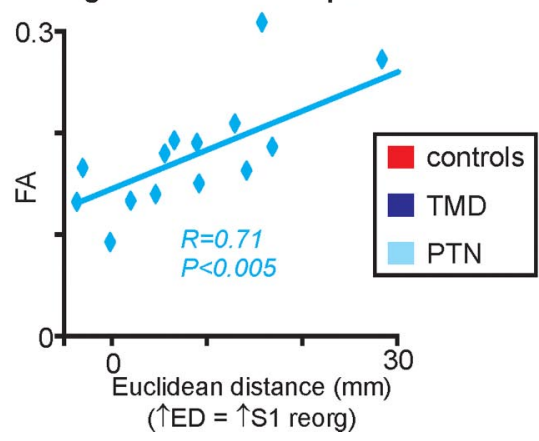

Figure 5. S1 anatomy and chronic pain. $A$, Overlays showing areas in which FA was altered in patients with painful TMD and PTN. Axial slice locations in MNI space are indicated at the top right of each image. Note that, whereas TMD patients display no significant change in the anatomy of the contralateral S1, PTN patients display a significant reduction in FA, an index of microstructure, in the region of $\mathrm{S} 1$ representing the lip. B, Overlays showing areas in which FA was significantly correlated with the degree of S1 reorganization in PTN patients (hot color scale). The plot of FA and degree of $\mathrm{S1}$ reorganization on the lower right shows this significant positive correlation. Also note that this significantly correlated region overlaps with the region activated by innocuous lip brushing (green shading). Further, the region in which baseline FA is significantly reduced in PTN patients (cool color scale) overlaps the region activated by lip brushing. The plot of mean ( \pm SEM) FA on the lower left shows that, in the region in which FA is reduced in PTN patients, FA is not altered in TMD patients.

neuropathic orofacial pain are associated with decreased S1 sensitivity to innocuous tactile stimuli. Others have reported similar decreases in S1 sensitivity to innocuous stimuli in patients with trigeminal neuropathic pain (Dancause et al., 2005), trigeminal neuralgia (Darian-Smith and Gilbert, 1995), and complex regional pain syndrome (Davis et al., 1998). It appears that chronic pain is associated with general reduction of S1 processing, indicating a long-term modulation of somatosensory function, even in patients who do not display changes in S1 organization, anatomy, or ongoing activity.

Several stages of cortical reorganization have been proposed. Initially, unmasking of normally inhibited connections may occur within minutes of the initial injury (Calford and Tweedale, 1988; Garraghty et al., 1991; Rossini et al., 1994) and result in small shifts in cortical organization $(<2-3 \mathrm{~mm})$.
Furthermore, particularly following deafferentation, S1 input may lessen as neuronal connections that no longer innervate tissue degenerate, resulting in a reduction in S1 blood flow. Subsequently, during a second, slower phase, structural changes, such as axonal sprouting, may occur, resulting in a significant shift in $\mathrm{S} 1$ functional organization in the order of $1-2 \mathrm{~cm}$. This may lead to a partial recovery of both blood flow and cortical volume, particularly in those individuals who develop pain. Indeed, our previous findings suggest long-term S1 graymatter volume recovery in individuals with complete spinal cord injury (Henderson et al., 2011). These series of changes could account for the CBF changes seen in the PTN patients in the present study and may account for the variations in baseline blood flow reported in numerous chronic pain investigations. 

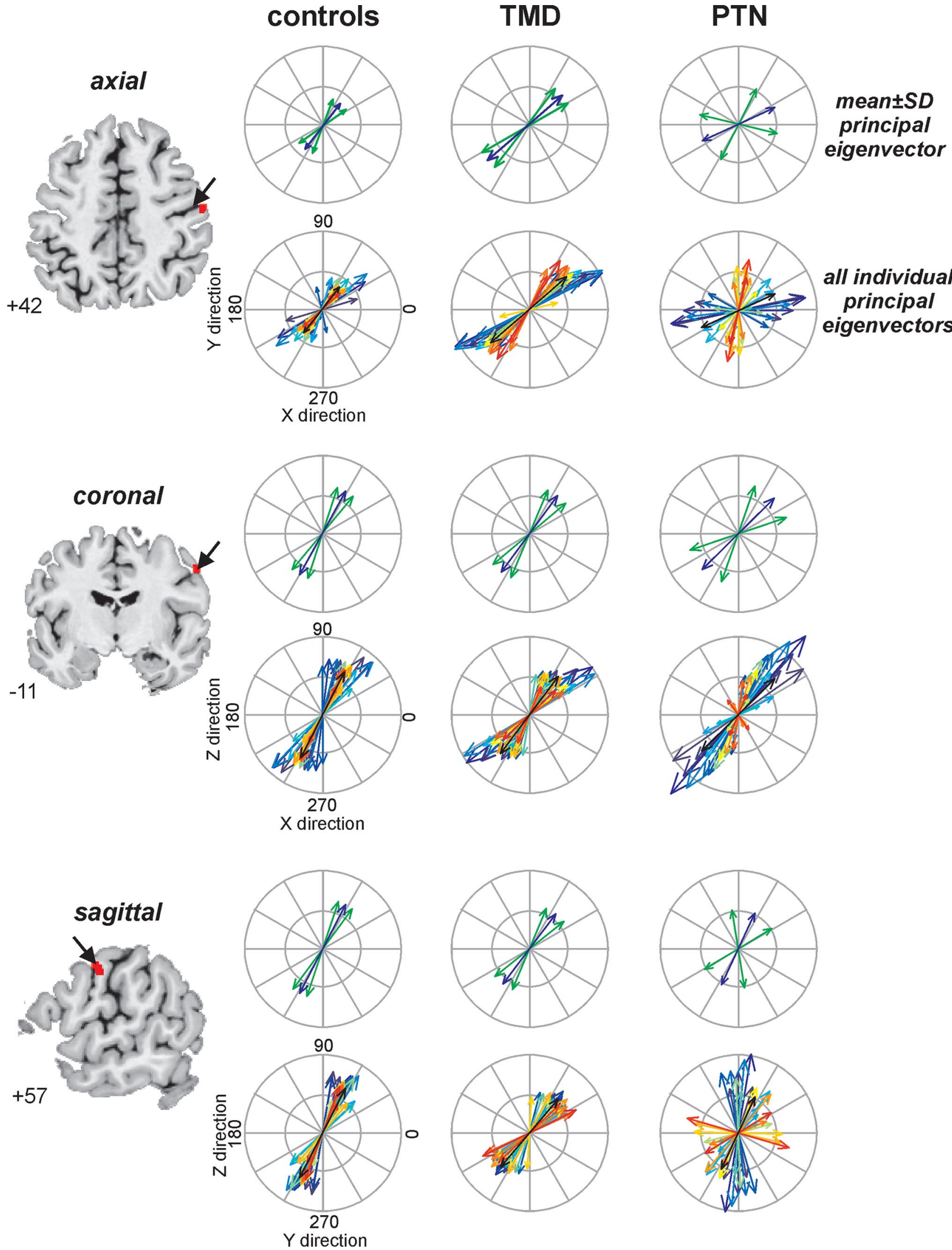

Figure 6. Principal eigenvectors associated with somatosensory cortex reorganization. Principal eigenvectorsin control, painful TMD, and PTN patients in the S1 region in which FA increases as $S 1$ reorganization increased (i.e., theregion representingthelip). Themean (blue) principal eigenvectors and median absolutedeviations (lightgreen)areshown inthetop panelfortheaxial, coronal, and sagittal planes. Thelowerpanel in the axial, coronal, and sagittal planes shows plots of individual eigenvectors color coded for direction. Note that, although the mean and individual eigenvectors are similar in control and TMD patients, in PTN patients the size and variation in direction are considerably different. That is, in all three planes, the variability of eigenvectors is considerably greater in PTN patients compared with controls and TMD patients. 
In addition, we found changes in S1 microstructure (i.e., FA) that were positively correlated to reorganization. FA is a measure of the direction and ease of water movement and, in white matter, increases as fibers become more uniform in their direction. In gray matter, FA can be affected by changes in neural organization, such as the ramification of dendrites or axon terminals, or nonneural changes, such as alterations in the vascular pattern or gliosis. Although the positive relationship between FA and S1 reorganization in PTN patients results from long-term changes in blood vessels or gliosis, it is also possible that this relationship results from neural changes, such as the expansion of cortical lateral connections, a similar result to that seen following complete spinal cord injury (Henderson et al., 2011). Evidence of axonal sprouting following injury exists in other sensory systems and even between sensory and motor systems (Darian-Smith and Gilbert, 1994, 1995; Dancause et al., 2005). Furthermore, plots of the tissue directionality within the lip region revealed that, although the mean and individual eigenvectors are similar in control and TMD patients, in PTN patients the size and variation in direction are considerably different. These data may result from the growth of lateral connections, although non-neural elements may also have an effect. We previously found similar diffusion changes in patients with complete spinal cord injuries (Henderson et al., 2011).

It is also possible that subcortical structures may play a significant role in S1 reorganization. Thalamic reorganization occurs following sectioning of peripheral afferents (Garraghty et al., 1991), sensory thalamus stump representations expand in amputees (Davis et al., 1998), and, following complete spinal cord injury, ventral thalamus cells that would normally respond to inputs from upper extremities respond to inputs from the neck and occiput (Lenz et al., 1987). Since neuropathic pain conditions are associated with changes in thalamic anatomy and biochemistry (Pattany et al., 2002; Gustin et al., 2010, 2011) and thalamic lesions often result in neuropathic pain (Klit et al., 2009), changes in thalamic function may result in neuropathic pain by altering firing patterns of thalamocortical loops (Sarnthein and Jeanmonod, 2008).

\section{Limitations}

It is possible that TMD is associated with $\mathrm{S} 1$ organizational, structural, or blood flow changes too subtle to be detected by our MRI techniques. Given the multiple preprocessing steps required for group MRI analysis, including the application of Gaussian filters, it is possible that changes in the order of millimeters could have gone undetected in TMD patients. Indeed, it appears that TMD is associated with some form of $\mathrm{S} 1$ alteration given that, along with PTN patients, $\mathrm{S} 1$ in TMD patients displayed decreased sensitivity to innocuous brushing compared with pain-free controls.

A number of our orofacial pain patients also described pain in other body regions. It is possible that these pains also affected our results, although one could argue that, because TMD patients had more widespread pain that often included the neck, the S1 region representing the neck should have shown even greater functional reorganization. In addition, although there was no significant difference in pain durations, TMD patients on average had their pain for a greater duration than PTN patients, which may have affected our results. Although again one could speculate that the TMD patients should have greater functional reorganization if chronicity per se was the cause of such alterations in cortical structure.

\section{Conclusions}

Our data have revealed that, while patients with neuropathic pain display cortical reorganization and associated changes in somatosensory cortex activity and anatomy, patients with nonneuropathic chronic pain do not. These data, by showing that pain per se is not associated with cortical plasticity, suggest that treatments aimed at reversing cortical reorganization should only be considered for use in patients with certain types of chronic pain.

\section{References}

Barker KL, Elliott CJ, Sackley CM, Fairbank JC (2008) Treatment of chronic back pain by sensory discrimination training. A Phase I RCT of a novel device (FairMed) vs. TENS. BMC Musculoskelet Disord 9:97.

Basser PJ, Pierpaoli C (1996) Microstructural and physiological features of tissues elucidated by quantitative-diffusion-tensor MRI. J Magn Reson B 111:209-219.

Birbaumer N, Lutzenberger W, Montoya P, Larbig W, Unertl K, Topfner S, Grodd W, Taub E, Flor H (1997) Effects of regional anesthesia on phantom limb pain are mirrored in changes in cortical reorganization. J Neurosci 17:5503-5508.

Calford MB, Tweedale R (1988) Immediate and chronic changes in responses of somatosensory cortex in adult flying-fox after digit amputation. Nature 332:446-448.

Dancause N, Barbay S, Frost SB, Plautz EJ, Chen D, Zoubina EV, Stowe AM, Nudo RJ (2005) Extensive cortical rewiring after brain injury. J Neurosci 25:10167-10179.

Darian-Smith C, Gilbert CD (1994) Axonal sprouting accompanies functional reorganization in adult cat striate cortex. Nature 368:737-740.

Darian-Smith C, Gilbert CD (1995) Topographic reorganization in the striate cortex of the adult cat and monkey is cortically mediated. J Neurosci 15:1631-1647.

Davis KD, Kiss ZH, Luo L, Tasker RR, Lozano AM, Dostrovsky JO (1998) Phantom sensations generated by thalamic microstimulation. Nature 391:385-387.

Dworkin SF, LeResche L (1992) Research diagnostic criteria for temporomandibular disorders: review, criteria, examinations and specifications, critique. J Craniomandib Disord 6:301-355.

Flor H, Elbert T, Knecht S, Wienbruch C, Pantev C, Birbaumer N, Larbig W, Taub E (1995) Phantom-limb pain as a perceptual correlate of cortical reorganization following arm amputation. Nature 375:482-484.

Flor H, Braun C, Elbert T, Birbaumer N (1997) Extensive reorganization of primary somatosensory cortex in chronic back pain patients. Neurosci Lett 224:5-8.

Friston KJ, Holmes AP, Worsley KP, Proline JB, Frith CD, Frackowiak RSJ (1995) Statistical parametric maps in functional imaging: a general imaging approach. J Hum Brain Mapp 2:189-210.

Garraghty PE, LaChica EA, Kaas JH (1991) Injury-induced reorganization of somatosensory cortex is accompanied by reductions in GABA staining. Somatosens Mot Res 8:347-354.

Gustin SM, Wrigley PJ, Siddall PJ, Henderson LA (2010) Brain Anatomy Changes Associated with Persistent Neuropathic Pain Following Spinal Cord Injury. Cereb Cortex 20:1409-1419.

Gustin SM, Peck CC, Wilcox SL, Nash PG, Murray GM, Henderson LA (2011) Different pain, different brain: thalamic anatomy in neuropathic and non-neuropathic chronic pain syndromes. J Neurosci 31:5956-5964.

Henderson LA, Gustin SM, Macey PM, Wrigley PJ, Siddall PJ (2011) Functional reorganization of the brain in humans following spinal cord injury: evidence for underlying changes in cortical anatomy. J Neurosci 31:26302637.

Hodzic A, Veit R, Karim AA, Erb M, Godde B (2004) Improvement and decline in tactile discrimination behavior after cortical plasticity induced by passive tactile coactivation. J Neurosci 24:442-446.

Kaas JH (1991) Plasticity of sensory and motor maps in adult mammals. Annu Rev Neurosci 14:137-167.

Klit H, Finnerup NB, Jensen TS (2009) Central post-stroke pain: clinical characteristics, pathophysiology, and management. Lancet Neurol 8:857868 .

Klug S, Anderer P, Saletu-Zyhlarz G, Freidl M, Marion F, Saletu B, Prause W, Aigner M (2011) Dysfunctional pain modulation in somatoform pain disorder patients. Eur Arch Psychiatry Clin Neurosci 261:267-275. 
Lenz FA, Tasker RR, Dostrovsky JO, Kwan HC, Gorecki J, Hirayama T, Murphy JT (1987) Abnormal single-unit activity recorded in the somatosensory thalamus of a quadriplegic patient with central pain. Pain 31:225-236.

Macey PM, Macey KE, Kumar R, Harper RM (2004) A method for removal of global effects from fMRI time series. Neuroimage 22:360-366.

Maihöfner C, Handwerker HO, Neundörfer B, Birklein F (2003) Patterns of cortical reorganization in complex regional pain syndrome. Neurology 61:1707-1715.

Manfredini D, Nardini LG (2010) TMD classification and epidemiology. In: Current concepts on temporomandibular disorders (Manfredini D, ed), pp 25-39. London: Quintessence.

Melzack R (1975) The McGill Pain Questionnaire: major properties and scoring methods. Pain 1:277-299.

Merzenich MM, Nelson RJ, Stryker MP, Cynader MS, Schoppmann A, Zook JM (1984) Somatosensory cortical map changes following digit amputation in adult monkeys. J Comp Neurol 224:591-605.

Moseley GL, Flor H (2012) Targeting cortical representations in the treatment of chronic pain: a review. Neurorehabil Neural Repair 26:646-652.

Napadow V, Kettner N, Ryan A, Kwong KK, Audette J, Hui KK (2006) Somatosensory cortical plasticity in carpal tunnel syndrome- a crosssectional fMRI evaluation. Neuroimage 31:520-530.

Nurmikko TJ, Eldridge PR (2001) Trigeminal neuralgia—pathophysiology, diagnosis and current treatment. Br J Anaesth 87:117-132

Pattany PM, Yezierski RP, Widerström-Noga EG, Bowen BC, MartinezArizala A, Garcia BR, Quencer RM (2002) Proton magnetic resonance spectroscopy of the thalamus in patients with chronic neuropathic pain after spinal cord injury. AJNR Am J Neuroradiol 23:901-905.

Petersen ET, Lim T, Golay X (2006) Model-free arterial spin labeling quantification approach for perfusion MRI. Magn Reson Med 55:219-232.

Petersen ET, Mouridsen K, Golay X, Golay X (2010) The QUASAR reproducibility study, part II: results from a multi-center arterial spin labeling test-retest study. Neuroimage 49:104-113.
Pleger B, Tegenthoff M, Schwenkreis P, Janssen F, Ragert P, Dinse HR, Völker B, Zenz M, Maier C (2004) Mean sustained pain levels are linked to hemispherical side-to-side differences of primary somatosensory cortex in the complex regional pain syndrome I. Exp Brain Res 155:115-119.

Rampon C, Jiang CH, Dong H, Tang YP, Lockhart DJ, Schultz PG, Tsien JZ, Hu Y (2000) Effects of environmental enrichment on gene expression in the brain. Proc Natl Acad Sci U S A 97:12880-12884.

Ro JY (2008) Functional role of peripheral glutamate receptors in craniofacial muscle pain and hyperalgesia. In: Fundamentals of musculoskeletal pain (Graven-Nielsen T, Arendt-Nielsen L, Mense S, eds), pp 33-45. Seattle: IASP.

Rossini PM, Martino G, Narici L, Pasquarelli A, Peresson M, Pizzella V, Tecchio F, Torrioli G, Romani GL (1994) Short-term brain 'plasticity' in humans: transient finger representation changes in sensory cortex somatotopy following ischemic anesthesia. Brain Res 642:169-177.

Sarlani E, Greenspan JD (2005) Why look in the brain for answers to temporomandibular disorder pain? Cells Tissues Organs 180:69-75.

Sarnthein J, Jeanmonod D (2008) High thalamocortical theta coherence in patients with neurogenic pain. Neuroimage 39:1910-1917.

Sörös P, Knecht S, Bantel C, Imai T, Wüsten R, Pantev C, Lütkenhöner B, Bürkle H, Henningsen H (2001) Functional reorganization of the human primary somatosensory cortex after acute pain demonstrated by magnetoencephalography. Neurosci Lett 298:195-198.

van Praag H, Kempermann G, Gage FH (2000) Neural consequences of environmental enrichment. Nat Rev Neurosci 1:191-198.

Vartiainen N, Kirveskari E, Kallio-Laine K, Kalso E, Forss N (2009) Cortical reorganization in primary somatosensory cortex in patients with unilateral chronic pain. J Pain 10:854-859.

Wrigley PJ, Press SR, Gustin SM, Macefield VG, Gandevia SC, Cousins MJ, Middleton JW, Henderson LA, Siddall PJ (2009) Neuropathic pain and primary somatosensory cortex reorganization following spinal cord injury. Pain 141:52-59. 